\title{
Inverse backscattering problem for perturbations of biharmonic operator
}

\author{
Teemu Tyni* and Markus Harju \\ Department of Mathematical Sciences \\ University of Oulu, Finland
}

\begin{abstract}
We consider the inverse backscattering problem for a biharmonic operator with two lower order perturbations in two and three dimensions. The inverse Born approximation is used to recover jumps and singularities of an unknown combination of potentials. Numerical examples are given to illustrate the practical usefulness of the method.
\end{abstract}

\section{Introduction}

This work is devoted to inverse backscattering problem for the perturbed biharmonic operator

$$
H_{4} u=\Delta^{2} u+\vec{q} \cdot \nabla u+V u,
$$

where $\Delta$ is the Laplacian and $\cdot$ denotes the dot-product. The bi-Laplacian is perturbed by first and zero order perturbations $\vec{q}$ and $V$ which we allow to be complexvalued and which will be specified below. We are looking for solutions to the scattering problem

$$
H_{4} u=k^{4} u, \quad u=u_{0}+u_{\mathrm{sc}}, \quad u_{0}(x, k, \theta)=\mathrm{e}^{\mathrm{i} k(x, \theta)}
$$

which are outgoing in the sense that they satisfy an analogue of Sommerfeld's radiation condition at the infinity [24]. Here $\theta \in \mathbb{S}^{n-1}=\left\{x \in \mathbb{R}^{n}:|x|=1\right\}$ and $k \in \mathbb{R}$ are the angle and wavenumber of the incident wave, respectively.

Our interest in scattering problems for (1) stems from the corresponding problems for the Schrödinger operator and the magnetic Schrödinger operator. Indeed,

*corresponding author, Teemu.Tyni@oulu.fi 
the latter operators have received quite extensive interest in the frame of inverse scattering. To this end, we refer to the works $[3,4,5,13,14,15,16,17,18,19,21,22]$ and the references therein. The main idea in these works is to extract information about the unknown potentials from the knowledge of scattering amplitude. Most prominently, limited scattering data is considered in the form of fixed energy, fixed angle or backscattering data.

Higher order operators can be met, for example, in the theory of vibrations of beams and study of elasticity [8]. Biharmonic operators, such as (1), are not so familiar in the inverse problems literature but they have recently gained attention from several authors. In [2] the following boundary value problem

$$
\left\{\begin{array}{l}
\left(\Delta^{2}+q\right) u=0, \text { in } \Omega, \\
u=f, \Delta u=g, \text { on } \partial \Omega
\end{array}\right.
$$

for the biharmonic operator with Navier boundary conditions was studied. They consider the recovery of $q$ from partial boundary data (Dirichlet-to-Neumann map) and proceed to prove certain stability estimates for this problem. In [25] the goal of the problem is the same as in the present paper: to recover both $\vec{q}$ and $V$ in $H_{4}$, however from partial Cauchy data on some subset of $\mathbb{R}^{n}$.

In terms of scattering problems for higher order operators we are only aware of $[1,11,23,24]$. This work is a continuation of [24] and a multidimensional counterpart of [23]. We investigate the inverse backscattering problem for (1), which means that our data is limited to measurement angles which are opposite to the incident angle. By considering the backscattering amplitude we define the inverse backscattering Born approximation and prove that it recovers jumps and singularities of a potential combination. As is always the case, the main difficulty in this approach is to obtain good enough estimates for the first nonlinear term in the backscattering Born series.

Next we recall the main ingredients about direct scattering for (1) from [24]. Let $H_{0}:=\Delta^{2}-k^{4}$. By using the equality $H_{0}=\left(-\Delta-k^{2}\right)\left(-\Delta+k^{2}\right)$ we see that $H_{0}$ consists of two parts; one corresponds to the Helmholtz operator and the other is non-singular. A fundamental solution to $H_{0}$ is given by

$$
G_{k}^{+}(|x|)=\frac{\mathrm{i}}{8 k^{2}}\left(\frac{|k|}{2 \pi|x|}\right)^{\frac{n-2}{2}}\left(H_{\frac{n-2}{2}}^{(1)}(|k||x|)+\frac{2 \mathrm{i}}{\pi} K_{\frac{n-2}{2}}(|k||x|)\right),
$$

where $H_{\frac{n-2}{2}}^{(1)}$ is the Hankel function of the first kind and $K_{\frac{n-2}{2}}$ is the Macdonald function, both of order $\frac{n-2}{2}$. By applying this fundamental solution to (1) we obtain so-called Lippmann-Schwinger integral equation

$$
u(x, k, \theta)=\mathrm{e}^{\mathrm{i} k(x, \theta)}-\int_{\mathbb{R}^{n}} G_{k}^{+}(|x-y|)[\vec{q}(y) \cdot \nabla u(y, k, \theta)+V(y) u(y, k, \theta)] \mathrm{d} y .
$$


The equivalence of (2) and (3) has been shown in [24]. If we denote the latter integral operator in (3) (including the minus sign) as $L_{k} u(x)$ the equation can be equivalently written as

$$
u_{\mathrm{sc}}(x)=\widetilde{u_{0}}+L_{k}\left(u_{\mathrm{sc}}\right), \quad \widetilde{u_{0}}:=L_{k} u_{0} .
$$

This integral equation can be solved by iterations defined as

$$
u_{j}(x, k, \theta)=L_{k}^{j} u_{0}(x, k, \theta), \quad j=0,1, \ldots
$$

Moreover, the following main results concerning the direct scattering problem are proved in [24].

Theorem 1.1. Let $\vec{q} \in W_{p, 2 \delta}^{1}\left(\mathbb{R}^{n}\right)$ and $V \in L_{2 \delta}^{p}\left(\mathbb{R}^{n}\right)$, where $n<p \leq \infty$ and $2 \delta>n-n / p$. Then there exists $k_{0}>1$ such that the function

$$
u_{\mathrm{sc}}(x, k, \theta)=\sum_{j=1}^{\infty} u_{j}(x, k, \theta)
$$

solves (4) uniquely in $H_{-\delta}^{2}\left(\mathbb{R}^{n}\right)$ for $k \geq k_{0}$.

Theorem 1.2. Let $\vec{q}$ and $V$ be as in Theorem 1.1. Then for $n=2,3$ and for fixed $k>0$ the solution $u(x, k, \theta)$ to (2) or (3) admits the representation

$u(x, k, \theta)=u_{0}(x, k, \theta)-\frac{\mathrm{i} \mathrm{e}^{-\mathrm{i}(n-1) \pi / 4}}{4(2 \pi)^{(n-1) / 2}} \frac{k^{(n-7) / 2} \mathrm{e}^{\mathrm{i} k|x|}}{|x|^{(n-1) / 2}} A\left(k, \theta, \theta^{\prime}\right)+o\left(|x|^{(1-n) / 2}\right), \quad|x| \rightarrow \infty$ where

$$
A\left(k, \theta, \theta^{\prime}\right)=\int_{\mathbb{R}^{n}} \mathrm{e}^{-\mathrm{i} k\left(\theta^{\prime}, y\right)}(\vec{q} \cdot \nabla u+V u) \mathrm{d} y
$$

and $\theta^{\prime}=x /|x|$. Theorem also holds in any dimension $n \geq 4$ if $\vec{q}$ and $V$ are compactly supported.

It was also shown that

$$
\left\|u_{j}\right\|_{H_{-\delta}^{1}\left(\mathbb{R}^{n}\right)} \leq C \frac{C_{0}^{j}}{k^{2 j-1}}, \quad j=1,2, \ldots
$$

for some constants $C, C_{0}>0$ depending on $n, p, \delta$ and the coefficients $\vec{q}$ and $V$ (see [24, proof of Corollary 4.3]).

This work is organized as follows. In Section 2 we define the inverse backscattering Born approximation and expand it in several terms. The smoothness of the remainder term $q_{\text {rest }}$ is analyzed in dimensions $n \geq 2$. Section 3 is devoted to the first nonlinear term $q_{1}$. We distinguish it in two terms and carry out the analysis of its smoothness in dimensions $n=2$ and $n=3$ separately. The reason for this is that we do not need to assume real-valuedness of the potentials in the two dimensional case. The main results from Section 2 and 3 are collected together in Section 4. Finally, in Section 5 we give numerical examples in two dimensions to illustrate the main results. 


\section{Backscattering Born approximation}

Let us start by fixing some notations. We define the Fourier transform of a Schwartz function $f$ as

$$
F(f)(\xi)=\widehat{f}(\xi):=(2 \pi)^{-\frac{n}{2}} \int_{\mathbb{R}^{n}} \mathrm{e}^{-\mathrm{i}(\xi, x)} f(x) \mathrm{d} x .
$$

The Fourier transform is extended to tempered distributions by duality. Let $1 \leq$ $p<\infty$ and $\delta \in \mathbb{R}$. We denote by $L_{\delta}^{p}\left(\mathbb{R}^{n}\right)$ the weighted Lebesgue spaces defined by finiteness of the norm

$$
\|f\|_{L_{\delta}^{p}\left(\mathbb{R}^{n}\right)}:=\left(\int_{\mathbb{R}^{n}}(1+|x|)^{\delta p}|f(x)|^{p} \mathrm{~d} x\right)^{\frac{1}{p}} .
$$

We say that $f$ belongs to the Sobolev space $W_{p, \delta}^{1}\left(\mathbb{R}^{n}\right)$ if $f, \nabla f \in L_{\delta}^{p}\left(\mathbb{R}^{n}\right)$. If $\vec{f}$ is vector valued, then we say that $\vec{f} \in W_{p, \delta}^{1}\left(\mathbb{R}^{n}\right)$, if each component of $\vec{f}$ belongs to the Sobolev space in the above sense. Similarly the vector valued Fourier transform $\widehat{\vec{f}}$ of $\vec{f}$ is to be understood coordinate-wise. As usual, we denote by $H^{s}\left(\mathbb{R}^{n}\right)$ the $L^{2}$-based Sobolev spaces, defined by the norm

$$
\|f\|_{H^{s}\left(\mathbb{R}^{n}\right)}:=\left(\int_{\mathbb{R}^{n}}\left(1+|x|^{2}\right)^{s}|\widehat{f}(x)|^{2} \mathrm{~d} x\right)^{\frac{1}{2}} .
$$

We denote the characteristic function of the interval $\left[-k_{0}, k_{0}\right]$ by $\chi(k)$. Finally, the letter $C$ will be used to denote a positive constant whose value can change from line to line.

Let us now turn to the backscattering problem and set $\theta^{\prime}=-\theta$. In this case the backscattering amplitude becomes

$$
A_{\mathrm{b}}(k, \theta):=A(k, \theta,-\theta)=\int_{\mathbb{R}^{n}} \mathrm{e}^{\mathrm{i} k(\theta, y)}[\vec{q}(y) \cdot \nabla u(y, k, \theta)+V(y) u(y, k, \theta)] \mathrm{d} y .
$$

For simplicity we set $A(k, \theta,-\theta)=0$ when $0 \leq k<k_{0}$, where $k_{0}>0$ can be chosen so large that that all the relevant quantities are well-defined and unique. If we substitute here $u_{0}$ instead of $u$ then we have the approximation

$$
\begin{aligned}
A_{\mathrm{b}}(k, \theta) \approx A_{0}(k, \theta) & :=\int_{\mathbb{R}^{n}} \mathrm{e}^{2 \mathrm{i} k(\theta, y)}[\mathrm{i} k \theta \cdot \vec{q}(y)+V(y)] \mathrm{d} y \\
& =\int_{\mathbb{R}^{n}} \mathrm{e}^{2 \mathrm{i} k(\theta, y)}\left[-\frac{1}{2} \nabla \cdot \vec{q}(y)+V(y)\right] \mathrm{d} y \\
& =(2 \pi)^{\frac{n}{2}} F^{-1}(\beta)(2 k \theta),
\end{aligned}
$$


where we denote

$$
\beta(x):=-\frac{1}{2} \nabla \cdot \vec{q}(x)+V(x)
$$

and where the divergence theorem was applied to integrate by parts once in the first term.

The approximation (7) suggests us to define the inverse backscattering Born approximation $q_{\mathrm{B}}$ of the potential combination $\beta$ by

$$
q_{\mathrm{B}}(x):=\frac{1}{(2 \pi)^{n}} \int_{0}^{\infty} k^{n-1} \int_{\mathbb{S}^{n-1}} \mathrm{e}^{-\mathrm{i} k(\theta, x)} A_{\mathrm{b}}\left(\frac{k}{2}, \theta\right) \mathrm{d} \theta \mathrm{d} k
$$

in the sense of distributions.

In view of the series representation (5) it is possible to expand

$$
A_{\mathrm{b}}(k, \theta)=\sum_{j=0}^{\infty} A_{j}(k, \theta),
$$

where

$$
A_{j}(k, \theta):=\int_{\mathbb{R}^{n}} \mathrm{e}^{\mathrm{i} k(\theta, y)}\left[\vec{q}(y) \cdot \nabla u_{j}(y, k, \theta)+V(y) u_{j}(y, k, \theta)\right] \mathrm{d} y, \quad j=0,1, \ldots
$$

Note that $A_{\mathrm{b}}$ only depends on the product $k \theta$. Moreover, we can write $q_{\mathrm{B}}=q_{0}+$ $q_{1}+q_{\text {rest }}$, where $q_{\text {rest }}:=\sum_{j=2}^{\infty} q_{j}$ and

$$
q_{j}(x):=\frac{1}{(2 \pi)^{n}} \int_{0}^{\infty} k^{n-1} \int_{\mathbb{S}^{n-1}} \mathrm{e}^{-\mathrm{i} k(\theta, x)} A_{j}\left(\frac{k}{2}, \theta\right) \mathrm{d} \theta \mathrm{d} k, \quad j=0,1, \ldots
$$

It is straight-forward to confirm that $q_{0}=\beta+\widetilde{q}$, where

$$
\widetilde{q}(x):=\frac{1}{(2 \pi)^{n}} \int_{0}^{2 k_{0}} k^{n-1} \int_{\mathbb{S}^{n-1}} \mathrm{e}^{-\mathrm{i} k(\theta, x)} \int_{\mathbb{R}^{n}} \mathrm{e}^{\mathrm{i} k(\theta, y)} \beta(y) \mathrm{d} y \mathrm{~d} \theta \mathrm{d} k \in C^{\infty}\left(\mathbb{R}^{n}\right) .
$$

In what follows we consider the remaining terms $q_{1}$ and $q_{\text {rest }}$. Our aim is to prove that they are more regular than $\beta$ itself is.

Lemma 2.1. Let $n \geq 2$ and $\vec{q}$ and $V$ be as in Theorem 1.1. Then $q_{\text {rest }} \in H^{s}\left(\mathbb{R}^{n}\right)$ for any $s<\frac{6-n}{2}$.

Proof. We start by estimating $A_{\text {rest }}(k, \theta):=\sum_{j=2}^{\infty} A_{j}(k, \theta)$. For $k>k_{0}$ we have by Cauchy-Schwarz inequality that

$$
\begin{aligned}
\left|A_{\text {rest }}(k, \theta)\right| & \leq \sum_{j=2}^{\infty} \int_{\mathbb{R}^{n}}\left[|\vec{q}|\left|\nabla u_{j}\right|+\left|V \| u_{j}\right|\right] \mathrm{d} y \\
& \leq \sum_{j=2}^{\infty}\left[\|\vec{q}\|_{L_{\delta}^{2}\left(\mathbb{R}^{n}\right)}\left\|\nabla u_{j}\right\|_{L_{-\delta}^{2}\left(\mathbb{R}^{n}\right)}+\|V\|_{L_{\delta}^{2}\left(\mathbb{R}^{n}\right)}\left\|u_{j}\right\|_{L_{-\delta}^{2}\left(\mathbb{R}^{n}\right)}\right] \leq \frac{C}{k^{3}},
\end{aligned}
$$


where we applied (6) and estimated the emerging geometric series. Then Fourier transform of $q_{\text {rest }}$ is estimated by $\left|\widehat{q_{\text {rest }}}(k \theta)\right| \leq C \chi(k / 2) k^{-3}$. It means that

$$
\left\|q_{\text {rest }}\right\|_{H^{s}\left(\mathbb{R}^{n}\right)}^{2}=\int_{0}^{\infty} k^{n-1} \int_{\mathbb{S}^{n-1}}\left(1+k^{2}\right)^{s}\left|\widehat{q_{\text {rest }}}(k \theta)\right|^{2} \mathrm{~d} \theta \mathrm{d} k \leq C \int_{2 k_{0}}^{\infty} k^{n-1} k^{2 s-6} \mathrm{~d} k,
$$

where the last integral converges if and only if $s<\frac{6-n}{2}$.

\section{Analysis of the first nonlinear term}

In this section we perform the more delicate investigation of the first non-linear term $q_{1}$. Most of the following results hold in any dimensions $n \geq 2$, but our main results have their useful range of applications in dimensions $n=2,3$. Let us introduce a shorthand notation

$$
\widetilde{V}:=\nabla \cdot \vec{q}-V .
$$

As $q_{1}$ is given by (9) we study $A_{1}$ first. By integrating by parts we see that it can be expanded as

$$
\begin{aligned}
A_{1}(k / 2, \theta)=-\int_{\mathbb{R}^{n}} \frac{\mathrm{i} k \theta}{2} \cdot \vec{q}(y) \mathrm{e}^{\mathrm{i} \frac{k}{2}(\theta, y)} u_{1}(y, k / 2, \theta) \mathrm{d} y & \\
& -\int_{\mathbb{R}^{n}} \widetilde{V}(y) \mathrm{e}^{\mathrm{i} \frac{k}{2}(\theta, y)} u_{1}(y, k / 2, \theta) \mathrm{d} y .
\end{aligned}
$$

Since

$$
u_{1}(y, k, \theta)=-\int_{\mathbb{R}^{n}} G_{k}^{+}(|y-z|)[\mathrm{i} k \theta \cdot \vec{q}(z)+V(z)] \mathrm{e}^{\mathrm{i} k(\theta, z)} \mathrm{d} z
$$

then by the symmetry in variables of integration we have

$$
\begin{aligned}
& A_{1}(k / 2, \theta)=\int_{\mathbb{R}^{n}} \int_{\mathbb{R}^{n}} G_{k / 2}^{+}(|y-z|) \mathrm{e}^{\mathrm{i} \frac{k}{2}(\theta, y+z)} {\left[\frac{\mathrm{i} k \theta \cdot \vec{q}(y)}{2} \frac{\mathrm{i} k \theta \cdot \vec{q}(z)}{2}\right.} \\
&\left.+\nabla \cdot \vec{q}(y) \frac{\mathrm{i} k \theta \cdot \vec{q}(z)}{2}+\widetilde{V}(y) V(z)\right] \mathrm{d} z \mathrm{~d} y .
\end{aligned}
$$

Let us denote

$$
G_{k}^{+}(|x|)=: G_{k}^{\mathrm{M}}(|x|)+G_{k}^{\mathrm{E}}(|x|),
$$

where $G_{k}^{\mathrm{M}}$ is the oscillating main part corresponding to Hankel function and the exponentially decaying part $G_{k}^{\mathrm{E}}$ corresponds to Macdonald function. It means that 
we can separate $A_{1}$ into two parts

$$
\begin{aligned}
A_{1}(k / 2, \theta) & =\int_{\mathbb{R}^{n}} \int_{\mathbb{R}^{n}} G_{k / 2}^{\mathrm{M}}(|y-z|) \mathrm{e}^{\mathrm{i} \frac{k}{2}(\theta, y+z)} \\
& \times\left[\frac{\mathrm{i} k \theta \cdot \vec{q}(y)}{2} \frac{\mathrm{i} k \theta \cdot \vec{q}(z)}{2}+\nabla \cdot \vec{q}(y) \frac{\mathrm{i} k \theta \cdot \vec{q}(z)}{2}+\widetilde{V}(y) V(z)\right] \mathrm{d} z \mathrm{~d} y \\
& \quad+\int_{\mathbb{R}^{n}} \int_{\mathbb{R}^{n}} G_{k / 2}^{\mathrm{E}}(|y-z|) \mathrm{e}^{\mathrm{i} \frac{k}{2}(\theta, y+z)} \\
& \times\left[\frac{\mathrm{i} k \theta \cdot \vec{q}(y)}{2} \frac{\mathrm{i} k \theta \cdot \vec{q}(z)}{2}+\nabla \cdot \vec{q}(y) \frac{\mathrm{i} k \theta \cdot \vec{q}(z)}{2}+\widetilde{V}(y) V(z)\right] \mathrm{d} z \mathrm{~d} y \\
= & : A_{\mathrm{M}}(k / 2, \theta)+A_{\mathrm{E}}(k / 2, \theta) .
\end{aligned}
$$

In view of this splitting we write $q_{1}$ as

$$
\begin{aligned}
q_{1}(x) & :=\frac{1}{(2 \pi)^{n}} \int_{0}^{\infty} k^{n-1} \chi(k / 2) \int_{\mathbb{S}^{n-1}} \mathrm{e}^{-\mathrm{i} k(\theta, x)} A_{\mathrm{M}}(k / 2, \theta) \mathrm{d} \theta \mathrm{d} k \\
& +\frac{1}{(2 \pi)^{n}} \int_{0}^{\infty} k^{n-1} \chi(k / 2) \int_{\mathbb{S}^{n-1}} \mathrm{e}^{-\mathrm{i} k(\theta, x)} A_{\mathrm{E}}(k / 2, \theta) \mathrm{d} \theta \mathrm{d} k=: q_{1, \mathrm{M}}(x)+q_{1, \mathrm{E}}(x) .
\end{aligned}
$$

First we analyze the latter part which has better behaviour.

Lemma 3.1. Let $n \geq 2, \vec{q}$ and $V$ be as in Theorem 1.1. Then $q_{1, \mathrm{E}} \in H^{s}\left(\mathbb{R}^{n}\right)$ for any $s<\frac{8-n}{2}$.

Proof. We will show that the Fourier transform of $q_{1, \mathrm{E}}$ belongs to $L_{s}^{2}\left(\mathbb{R}^{n}\right)$. To do this, we divide $A_{\mathrm{E}}$ into three parts

$$
\begin{aligned}
A_{\mathrm{E}}(k / 2, \theta) & =\int_{\mathbb{R}^{n}} \int_{\mathbb{R}^{n}} G_{k / 2}^{\mathrm{E}}(|y-z|) \mathrm{e}^{\mathrm{i} \frac{k}{2}(\theta, y+z)} \\
\times\left[\frac{\mathrm{i} k \theta \cdot \vec{q}(y)}{2} \frac{\mathrm{i} k \theta \cdot \vec{q}(z)}{2}+\nabla \cdot \vec{q}(y) \frac{\mathrm{i} k \theta \cdot \vec{q}(z)}{2}+\widetilde{V}(y) V(z)\right] \mathrm{d} z \mathrm{~d} y & =: A_{\mathrm{E}}^{(1)}+A_{\mathrm{E}}^{(2)}+A_{\mathrm{E}}^{(3)} .
\end{aligned}
$$

We can interpret the double integral appearing in these formulas as a $2 n$-dimensional convolution at $(0,0) \in \mathbb{R}^{n} \times \mathbb{R}^{n}$. Therefore, let us consider

$$
T(f, g):=\left(G_{k / 2}^{\mathrm{E}}(|y-z|) \mathrm{e}^{-\mathrm{i} \frac{k}{2}(\theta, y+z)} *(f \otimes g)\right)(0,0)
$$

formally for some functions $f$ and $g$.

For the $2 n$-dimensional Fourier transform $\mathcal{F}$ we have $\mathcal{F}(\psi * \varphi)=(2 \pi)^{n} \mathcal{F} \psi \mathcal{F} \varphi$ and so

$$
T(f, g)=(2 \pi)^{n} \mathcal{F}^{-1}\left(\mathcal{F}\left(G_{k / 2}^{\mathrm{E}}(|y-z|) \mathrm{e}^{-\mathrm{i} \frac{k}{2}(\theta, y+z)}\right)(\eta, \mu) \widehat{f}(\eta) \widehat{g}(\mu)\right)(0,0) .
$$


Because for $k>0$ we have

$$
\left.\widehat{G_{k}^{\mathrm{E}}(|x|}\right)(\xi)=\frac{1}{2 k^{2}} \frac{1}{|\xi|^{2}+k^{2}}
$$

then we may calculate

$$
\begin{aligned}
\mathcal{F}\left(G_{k / 2}^{\mathrm{E}}(|y-z|) \mathrm{e}^{-\mathrm{i} \frac{k}{2}(\theta, y+z)}\right)(\eta, \mu) & \\
=(2 \pi)^{-n} & \int_{\mathbb{R}^{n}} \int_{\mathbb{R}^{n}} \mathrm{e}^{-\mathrm{i}(\eta, y)} \mathrm{e}^{-\mathrm{i}(\mu, z)} G_{k / 2}^{\mathrm{E}}(|y-z|) \mathrm{e}^{-\mathrm{i} \frac{k}{2}(\theta, y+z)} \mathrm{d} y \mathrm{~d} z \\
= & \frac{2(2 \pi)^{-\frac{n}{2}}}{k^{2}} \int_{\mathbb{R}^{n}} \frac{\mathrm{e}^{-\mathrm{i}(z, \eta+\mu+k \theta)}}{\left|\eta+\frac{k \theta}{2}\right|^{2}+k^{2} / 4} \mathrm{~d} z=\frac{2(2 \pi)^{\frac{n}{2}}}{k^{2}} \frac{\delta_{0}(\eta+\mu+k \theta)}{\left|\eta+\frac{k \theta}{2}\right|^{2}+k^{2} / 4}
\end{aligned}
$$

in the sense of distributions. Here $\delta_{0}$ is the Dirac delta distribution. This means that

$$
\begin{aligned}
T(f, g) & =\int_{\mathbb{R}^{n}} \int_{\mathbb{R}^{n}} \frac{2(2 \pi)^{\frac{n}{2}}}{k^{2}} \frac{\delta_{0}(\eta+\mu+k \theta)}{\left|\eta+\frac{k \theta}{2}\right|^{2}+k^{2} / 4} \widehat{f}(\eta) \widehat{g}(\mu) \mathrm{d} \eta \mathrm{d} \mu \\
& =\frac{2(2 \pi)^{\frac{n}{2}}}{k^{2}} \int_{\mathbb{R}^{n}} \frac{\widehat{f}(-\mu-k \theta) \widehat{g}(\mu)}{\left|\mu+\frac{k \theta}{2}\right|^{2}+k^{2} / 4} \mathrm{~d} \mu \\
& =\frac{2(2 \pi)^{\frac{n}{2}}}{k^{2}} \int_{\mathbb{R}^{n}} \frac{\widehat{f}\left(-\mu-\frac{k \theta}{2}\right) \widehat{g}\left(\mu-\frac{k \theta}{2}\right)}{|\mu|^{2}+k^{2} / 4} \mathrm{~d} \mu,
\end{aligned}
$$

where we have changed the variables $\mu \mapsto \mu-\frac{k \theta}{2}$ in the last step. The above calculations give us a way to represent the terms of $A_{\mathrm{E}}$ as

$$
\begin{aligned}
& A_{\mathrm{E}}^{(1)}=\frac{2(2 \pi)^{\frac{n}{2}}}{k^{2}} \int_{\mathbb{R}^{n}} \frac{\frac{\mathrm{i} k \theta}{2} \cdot \widehat{\vec{q}}\left(-\mu-\frac{k \theta}{2}\right) \frac{\mathrm{i} k \theta}{2} \cdot \widehat{\vec{q}}\left(\mu-\frac{k \theta}{2}\right)}{|\mu|^{2}+k^{2} / 4} \mathrm{~d} \mu, \\
& A_{\mathrm{E}}^{(2)}=\frac{2(2 \pi)^{\frac{n}{2}}}{k^{2}} \int_{\mathbb{R}^{n}} \frac{\widehat{\nabla \cdot \vec{q}}\left(-\mu-\frac{k \theta}{2}\right) \frac{\mathrm{i} k \theta}{2} \cdot \widehat{\vec{q}}\left(\mu-\frac{k \theta}{2}\right)}{|\mu|^{2}+k^{2} / 4} \mathrm{~d} \mu
\end{aligned}
$$

and

$$
A_{\mathrm{E}}^{(3)}=\frac{2(2 \pi)^{\frac{n}{2}}}{k^{2}} \int_{\mathbb{R}^{n}} \frac{\widehat{\widetilde{V}}\left(-\mu-\frac{k \theta}{2}\right) \widehat{V}\left(\mu-\frac{k \theta}{2}\right)}{|\mu|^{2}+k^{2} / 4} \mathrm{~d} \mu .
$$

By integration by parts $\widehat{\nabla \cdot \vec{q}}(\xi)=\mathrm{i} \xi \cdot \widehat{\vec{q}}(\xi)$ and we have

$$
A_{\mathrm{E}}^{(1)}+A_{\mathrm{E}}^{(2)}=-\frac{2(2 \pi)^{\frac{n}{2}}}{k^{2}} \int_{\mathbb{R}^{n}} \frac{\mathrm{i} \mu \cdot \widehat{\vec{q}}\left(-\mu-\frac{k \theta}{2}\right) \frac{\mathrm{i} k \theta}{2} \cdot \widehat{\vec{q}}\left(\mu-\frac{k \theta}{2}\right)}{|\mu|^{2}+k^{2} / 4} \mathrm{~d} \mu .
$$


The part of $q_{1, \mathrm{E}}$ corresponding to $A_{\mathrm{E}}^{(1)}+A_{\mathrm{E}}^{(2)}$ is

$$
\begin{aligned}
& q_{1, \mathrm{E}}^{(1+2)}(x)= \\
& -\frac{2}{(2 \pi)^{\frac{n}{2}}} \int_{2 k_{0}}^{\infty} k^{n-1} \int_{\mathbb{S}^{n-1}} \mathrm{e}^{-\mathrm{i} k(\theta, x)} \frac{1}{k^{2}} \int_{\mathbb{R}^{n}} \frac{\mathrm{i} \mu \cdot \widehat{\vec{q}}\left(-\mu-\frac{k \theta}{2}\right) \frac{\mathrm{i} k \theta}{2} \cdot \widehat{\vec{q}}\left(\mu-\frac{k \theta}{2}\right)}{|\mu|^{2}+k^{2} / 4} \mathrm{~d} \mu \mathrm{d} \theta \mathrm{d} k \\
& \quad=-\frac{2}{(2 \pi)^{\frac{n}{2}}} \int_{|\xi|>2 k_{0}} \frac{1}{|\xi|^{2}} \mathrm{e}^{-\mathrm{i}(x, \xi)} \int_{\mathbb{R}^{n}} \frac{\mathrm{i} \mu \cdot \widehat{\vec{q}}\left(-\mu-\frac{\xi}{2}\right) \frac{\mathrm{i} \xi}{2} \cdot \widehat{\vec{q}}\left(\mu-\frac{\xi}{2}\right)}{|\mu|^{2}+|\xi|^{2} / 4} \mathrm{~d} \mu \mathrm{d} \xi .
\end{aligned}
$$

Then the Fourier transform of $q_{1, \mathrm{E}}^{(1+2)}$ is

$$
\widehat{q_{1, \mathrm{E}}^{(1+2)}}(\xi)=-\frac{\chi(|\xi| / 2)}{|\xi|^{2}} \int_{\mathbb{R}^{n}} \frac{\mathrm{i} \mu \cdot \widehat{\vec{q}}\left(-\mu-\frac{\xi}{2}\right) \mathrm{i} \xi \cdot \widehat{\vec{q}}\left(\mu-\frac{\xi}{2}\right)}{|\mu|^{2}+|\xi|^{2} / 4} \mathrm{~d} \mu .
$$

Following [18] we write here

$$
\begin{aligned}
\mathrm{i} \mu \cdot \widehat{\vec{q}}\left(-\mu-\frac{\xi}{2}\right) \mathrm{i} \xi \cdot \widehat{\vec{q}}\left(\mu-\frac{\xi}{2}\right)= & \frac{1}{2}\left[-\mathrm{i}\left(-\mu-\frac{\xi}{2}\right) \cdot \widehat{\vec{q}}\left(-\mu-\frac{\xi}{2}\right)+\mathrm{i}\left(\mu-\frac{\xi}{2}\right) \cdot \widehat{\vec{q}}\left(-\mu-\frac{\xi}{2}\right)\right] \\
& \times\left[-\mathrm{i}\left(\mu-\frac{\xi}{2}\right) \cdot \widehat{\vec{q}}\left(\mu-\frac{\xi}{2}\right)-\mathrm{i}\left(-\mu-\frac{\xi}{2}\right) \cdot \widehat{\vec{q}}\left(\mu-\frac{\xi}{2}\right)\right] .
\end{aligned}
$$

This allows us to integrate by parts and expand to obtain

$$
\begin{aligned}
\widehat{q_{1, \mathrm{E}}^{(1+2)}}(\xi) & =-\frac{\chi(|\xi| / 2)}{2|\xi|^{2}} \int_{\mathbb{R}^{n}} \frac{\widehat{\nabla \cdot \vec{q}}\left(-\mu-\frac{\xi}{2}\right) \widehat{\nabla \cdot \vec{q}}\left(\mu-\frac{\xi}{2}\right)}{|\mu|^{2}+|\xi|^{2} / 4} \mathrm{~d} \mu \\
& +\frac{\chi(|\xi| / 2)}{2|\xi|^{2}} \int_{\mathbb{R}^{n}} \frac{\sum_{j, k=1}^{n} \widehat{\partial_{j} q_{k}}\left(-\mu-\frac{\xi}{2}\right) \widehat{\partial_{k} q_{j}}\left(\mu-\frac{\xi}{2}\right)}{|\mu|^{2}+|\xi|^{2} / 4} \mathrm{~d} \mu .
\end{aligned}
$$

Now instead of combinations of $V$ and $\vec{q}$ it suffices to consider functions $f$ and $g$ from $L_{2 \delta}^{p}\left(\mathbb{R}^{n}\right)$. A simple application of Cauchy-Schwarz inequality and Parseval's equality gives

$$
\frac{\chi(|\xi| / 2)}{|\xi|^{2}}\left|\int_{\mathbb{R}^{n}} \frac{\widehat{f}\left(-\mu-\frac{\xi}{2}\right) \widehat{g}\left(\mu-\frac{\xi}{2}\right)}{|\mu|^{2}+|\xi|^{2} / 4} \mathrm{~d} \mu\right| \leq \frac{4 \chi(|\xi| / 2)}{|\xi|^{4}}\|f\|_{L^{2}(\mathbb{R})}\|g\|_{L^{2}(\mathbb{R})} .
$$

This means that

$$
\left\|\widehat{q_{1, \mathrm{E}}}\right\|_{L_{s}^{2}\left(\mathbb{R}^{n}\right)}^{2} \leq C \int_{|\xi|>2 k_{0}}(1+|\xi|)^{2 s} \frac{1}{|\xi|^{8}} \mathrm{~d} \xi
$$

and this integral converges if and only if $s<\frac{8-n}{2}$. 
Next we consider the more complicated part of $q_{1}$, namely, $q_{1, \mathrm{M}}$. In order to estimate the smoothness of this term we first derive an alternative form for it closely following the proof of Lemma 3.1.

Lemma 3.2. Let $n \geq 2$. If $\vec{q}$ and $V$ are as in Theorem 1.1, then

$$
\begin{aligned}
q_{1, \mathrm{M}}(x) & =\frac{(2 \pi)^{\frac{n}{2}}}{2} \mathcal{F}^{-1}\left(\frac{\chi(|\mu+\eta| / 2)}{|\mu+\eta|^{2}} \frac{\widehat{\nabla \cdot \vec{q}}(\eta) \widehat{\nabla \cdot \vec{q}}(\mu)}{(\mu, \eta)+\mathrm{i} 0}\right)(x, x) \\
& -\frac{(2 \pi)^{\frac{n}{2}}}{2} \mathcal{F}^{-1}\left(\frac{\chi(|\mu+\eta| / 2)}{|\mu+\eta|^{2}} \frac{\sum_{j, k=1}^{n} \widehat{\partial_{j} q_{k}}(\eta) \widehat{\partial_{k} q_{j}}(\mu)}{(\mu, \eta)+\mathrm{i} 0}\right)(x, x) \\
& -2(2 \pi)^{\frac{n}{2}} \mathcal{F}^{-1}\left(\frac{\chi(|\mu+\eta| / 2)}{|\mu+\eta|^{2}} \frac{\widehat{\widetilde{V}}(\eta) \widehat{V}(\mu)}{(\mu, \eta)+\mathrm{i} 0}\right)(x, x)
\end{aligned}
$$

in the sense of distributions. Here $\widehat{\cdot}$ denotes the Fourier transform in $n$ dimensions and $\mathcal{F}^{-1}$ is the $2 n$-dimensional inverse Fourier transform.

Proof. We split

$$
\begin{aligned}
& \quad q_{1, \mathrm{M}}(x)=\frac{1}{(2 \pi)^{n}} \int_{0}^{\infty} k^{n-1} \int_{\mathbb{S}^{n-1}} \mathrm{e}^{-\mathrm{i} k(\theta, x)} \int_{\mathbb{R}^{n}} \int_{\mathbb{R}^{n}} G_{k / 2}^{\mathrm{M}}(|y-z|) \mathrm{e}^{\mathrm{i} \frac{k}{2}(\theta, y+z)} \\
& \times\left[\frac{\mathrm{i} k \theta \cdot \vec{q}(y)}{2} \frac{\mathrm{i} k \theta \cdot \vec{q}(z)}{2}+\nabla \cdot \vec{q}(y) \frac{\mathrm{i} k \theta \cdot \vec{q}(z)}{2}+\widetilde{V}(y) V(z)\right] \mathrm{d} z \mathrm{~d} y \mathrm{~d} \theta \mathrm{d} k=: I_{1}+I_{2}+I_{3} .
\end{aligned}
$$

As in $[7,13]$ we start from the expression

$$
I_{3}=\int_{\mathbb{R}^{n} \times \mathbb{R}^{n}} \frac{1}{(2 \pi)^{n}} \int_{0}^{\infty} k^{n-1} \int_{\mathbb{S}^{n-1}} G_{k / 2}^{\mathrm{M}}(|y-z|) \mathrm{e}^{\mathrm{i} \frac{k}{2}(\theta, y+z-2 x)} \mathrm{d} \theta \mathrm{d} k \widetilde{V}(y) V(z) \mathrm{d} y \mathrm{~d} z,
$$

which can be understood as a $2 n$-dimensional convolution $I_{3}=(2 \pi)^{-n}(G *(\widetilde{V} \otimes$ $V))(x, x)$, where

$$
G(y, z):=\int_{0}^{\infty} k^{n-1} \chi(k / 2) \int_{\mathbb{S}^{n-1}} G_{k / 2}^{\mathrm{M}}(|y-z|) \mathrm{e}^{-\mathrm{i} \frac{k}{2}(\theta, y+z)} \mathrm{d} \theta \mathrm{d} k .
$$

To obtain the claimed formula we consider the $2 n$-dimensional Fourier transform of $G$ as

$$
\begin{aligned}
\mathcal{F} G(\eta, \mu)=(2 \pi)^{-n} \int_{\mathbb{R}^{n} \times \mathbb{R}^{n}} & \int_{0}^{\infty} k^{n-1} \chi(k / 2) \\
& \times \int_{\mathbb{S}^{n-1}} G_{k / 2}^{\mathrm{M}}(|y-z|) \mathrm{e}^{-\mathrm{i} \frac{k}{2}(\theta, y+z)} \mathrm{e}^{-\mathrm{i}(\eta, y)} \mathrm{e}^{-\mathrm{i}(\mu, z)} \mathrm{d} \theta \mathrm{d} k \mathrm{~d} y \mathrm{~d} z .
\end{aligned}
$$


Recall that the Fourier transform of $G_{k}^{\mathrm{M}}(|x|)$ is given by

$$
\frac{1}{2 k^{2}} \frac{1}{|\xi|^{2}-k^{2}-\mathrm{i} 0} \text {. }
$$

Hence, by the change of variables $y-z=\xi$, we obtain

$$
\begin{aligned}
\mathcal{F} G(\eta, \mu)= & (2 \pi)^{-n} \int_{0}^{\infty} k^{n-1} \chi(k / 2) \int_{\mathbb{S}^{n-1}} \int_{\mathbb{R}^{n}} G_{k / 2}^{\mathrm{M}}(|\xi|) \mathrm{e}^{\mathrm{i}\left(\frac{k \theta}{2}+\eta, \xi\right)} \\
& \times \int_{\mathbb{R}^{n}} \mathrm{e}^{-\mathrm{i}(k \theta+\mu+\eta, z)} \mathrm{d} z \mathrm{~d} \xi \mathrm{d} \theta \mathrm{d} k \\
= & 2(2 \pi)^{\frac{n}{2}} \int_{0}^{\infty} k^{n-3} \chi(k / 2) \int_{\mathbb{S}^{n-1}} \frac{\delta_{0}(k \theta+\mu+\eta)}{\left|\frac{k \theta}{2}+\eta\right|^{2}-\frac{k^{2}}{4}-\mathrm{i} 0} \mathrm{~d} \theta \mathrm{d} k \\
= & -2(2 \pi)^{\frac{n}{2}} \frac{\chi(|\mu+\eta| / 2)}{|\mu+\eta|^{2}} \frac{1}{(\mu, \eta)+\mathrm{i} 0}
\end{aligned}
$$

in the sense of distributions. Applying $\mathcal{F}(f * g)=(2 \pi)^{n} \mathcal{F} f \mathcal{F} g$ we obtain

$$
I_{3}=-2(2 \pi)^{\frac{n}{2}} \mathcal{F}^{-1}\left(\frac{\chi(|\mu+\eta| / 2)}{|\mu+\eta|^{2}} \frac{\widehat{\widetilde{V}}(\eta) \widehat{V}(\mu)}{(\mu, \eta)+\mathrm{i} 0}\right)(x, x) .
$$

Proceeding analogously for $I_{1}$ and $I_{2}$ and by using symmetry in $\eta$ and $\mu$ we may combine them as in the proof of Lemma 3.1 to obtain

$$
\begin{aligned}
I_{1}+I_{2}= & \frac{(2 \pi)^{\frac{n}{2}}}{2} \mathcal{F}^{-1}\left(\frac{\chi(|\mu+\eta| / 2)}{|\mu+\eta|^{2}} \frac{(\mu-\eta) \cdot \widehat{\vec{q}}(\eta)(\mu+\eta) \cdot \widehat{\vec{q}}(\mu)}{(\mu, \eta)+\mathrm{i} 0}\right)(x, x) \\
= & \frac{(2 \pi)^{\frac{n}{2}}}{2} \mathcal{F}^{-1}\left(\frac{\chi(|\mu+\eta| / 2)}{|\mu+\eta|^{2}} \frac{\widehat{\nabla \cdot \vec{q}}(\eta) \widehat{\nabla \cdot \vec{q}}(\mu)}{(\mu, \eta)+\mathrm{i} 0}\right)(x, x) \\
& -\frac{(2 \pi)^{\frac{n}{2}}}{2} \mathcal{F}^{-1}\left(\frac{\chi(|\mu+\eta| / 2)}{|\mu+\eta|^{2}} \frac{\sum_{j, k=1}^{n} \widehat{\partial_{j} q_{k}}(\eta) \widehat{\partial_{k} q_{j}}(\mu)}{(\mu, \eta)+\mathrm{i} 0}\right)(x, x)
\end{aligned}
$$

as claimed.

According to Lemma 3.2 it suffices to study the regularity of

$$
I=(2 \pi)^{n} \mathcal{F}^{-1}\left(\frac{\chi(|\mu+\eta| / 2)}{|\mu+\eta|^{2}} \frac{\widehat{f}(\eta) \widehat{g}(\mu)}{(\mu, \eta)+\mathrm{i} 0}\right)(x, x)
$$

for functions $f, g \in L_{2 \delta}^{p}(\mathbb{R}), n<p \leq \infty$ and $2 \delta>n-\frac{n}{p}$. We use Sokhotski-Plemelj formula (see e.g., [12])

$$
\frac{1}{x \pm \mathrm{i} 0}=\mathrm{p} \cdot \mathrm{v} \cdot \frac{1}{x} \mp \mathrm{i} \pi \delta_{0}
$$


to expand

$$
\begin{aligned}
I=\mathrm{p} \cdot \mathrm{v} & \int_{\mathbb{R}^{n} \times \mathbb{R}^{n}} \mathrm{e}^{\mathrm{i}(x, \eta+\mu)} \frac{\chi(|\mu+\eta| / 2)}{|\mu+\eta|^{2}} \frac{\widehat{f}(\eta) \widehat{g}(\mu)}{(\eta, \mu)} \mathrm{d} \eta \mathrm{d} \mu \\
& -\mathrm{i} \pi \int_{\mathbb{R}^{n} \times \mathbb{R}^{n}} \mathrm{e}^{\mathrm{i}(x, \eta+\mu)} \frac{\chi(|\mu+\eta| / 2)}{|\mu+\eta|^{2}} \widehat{f}(\eta) \widehat{g}(\mu) \delta_{0}((\eta, \mu)=0) \mathrm{d} \eta \mathrm{d} \mu=: I^{\prime}+I^{\prime \prime} .
\end{aligned}
$$

The analysis of the terms $I^{\prime}$ and $I^{\prime \prime}$ is next carried out separately in two and three dimensional cases.

\subsection{Two-dimensional case}

Lemma 3.3. Let $n=2$ and $f, g \in L_{2 \delta}^{p}\left(\mathbb{R}^{2}\right)$ with $2<p \leq \infty$ and $2 \delta>2-\frac{2}{p}$. Then $I^{\prime \prime}$ defines a bounded and continuous function.

Proof. We follow [13] and change variables $\mu=t \gamma$ to obtain

$$
I^{\prime \prime}=C \int_{\mathbb{R}^{2}} \int_{0}^{\infty} t \int_{\mathbb{S}^{1}} \mathrm{e}^{\mathrm{i}(x, \eta+t \gamma)} \frac{\chi(|\eta+t \gamma| / 2)}{|\eta+t \gamma|^{2}} \widehat{f}(\eta) \widehat{g}(t \gamma) \delta_{0}(t|\eta|(\eta /|\eta|, \gamma)=0) \mathrm{d} \gamma \mathrm{d} t \mathrm{~d} \eta .
$$

Since $\delta_{0}(\alpha H)=\frac{1}{\alpha} \delta_{0}(H)$ if $\alpha>0$ then we find that

$$
\begin{aligned}
I^{\prime \prime} & =C \int_{\mathbb{R}^{2}} \int_{-\infty}^{\infty} \mathrm{e}^{\mathrm{i}\left(x, \eta+t \eta^{\perp}\right)} \frac{\chi\left(\left|\eta+t \eta^{\perp}\right| / 2\right)}{\left|\eta+t \eta^{\perp}\right|^{2}} \widehat{f}(\eta) \widehat{g}\left(t \eta^{\perp}\right)|\eta|^{-1} \mathrm{~d} t \mathrm{~d} \eta \\
& =C \int_{0}^{\infty} \int_{\mathbb{S}^{1}} \int_{0}^{\infty} \mathrm{e}^{\mathrm{i}\left(x, r \gamma+t \gamma^{\perp}\right)} \frac{\chi\left(\left|r \gamma+t \gamma^{\perp}\right| / 2\right)}{\left|r \gamma+t \gamma^{\perp}\right|^{2}} \widehat{f}(r \gamma) \widehat{g}\left(r \gamma^{\perp}\right) \mathrm{d} t \mathrm{~d} \gamma \mathrm{d} r
\end{aligned}
$$

where $\eta^{\perp}$ is the unit vector perpendicular to $\eta$ chosen according to any specific orthogonal reference.

Note that $\left|r \gamma+t \gamma^{\perp}\right|^{2}=r^{2}+t^{2}$. Since $f$ and $g$ are integrable their Fourier transforms are bounded and therefore this integral has no singularities at origin. Hence, if we integrate over $\int_{0}^{1}$ for $t$ and/or $r$ the integral is bounded by the $L^{1}$ norms of $f$ and $g$. So it remains to consider the integral $\int_{1}^{\infty}$. By Cauchy-Schwarz inequality

$$
\begin{aligned}
\left|\int_{1}^{\infty} \int_{\mathbb{S}^{1}} \int_{1}^{\infty} \mathrm{e}^{\mathrm{i}\left(x, r \gamma+t \gamma^{\perp}\right)} \frac{\chi\left(\left|r \gamma+t \gamma^{\perp}\right| / 2\right)}{\left|r \gamma+t \gamma^{\perp}\right|^{2}} \widehat{f}(r \gamma) \widehat{g}\left(r \gamma^{\perp}\right) \mathrm{d} t \mathrm{~d} \gamma \mathrm{d} r\right| \\
\leq \int_{1}^{\infty} \int_{1}^{\infty}\left(\int_{\mathbb{S}^{1}} \frac{|\widehat{f}(r \gamma)|^{2}}{1+r^{2}} \mathrm{~d} \gamma\right)^{\frac{1}{2}}\left(\int_{\mathbb{S}^{1}} \frac{|\widehat{g}(t \gamma)|^{2}}{1+t^{2}} \mathrm{~d} \gamma\right)^{\frac{1}{2}} \mathrm{~d} t \mathrm{~d} r .
\end{aligned}
$$


The above integrals are well-defined because $\widehat{f}$ and $\widehat{g}$ are continuous. Now it suffices to estimate

$$
\begin{aligned}
\int_{1}^{\infty}\left(\int_{\mathbb{S}^{1}}\right. & \left.\frac{|f(r \gamma)|^{2}}{1+r^{2}} \mathrm{~d} \gamma\right)^{\frac{1}{2}} \mathrm{~d} r \\
& \leq\left(\int_{1}^{\infty} \frac{\mathrm{d} r}{\left(1+r^{2}\right) r}\right)^{\frac{1}{2}}\left(\int_{1}^{\infty} \int_{\mathbb{S}^{1}}\left(1+r^{2}\right) r \frac{|f(r \gamma)|^{2}}{1+r^{2}} \mathrm{~d} \gamma \mathrm{d} r\right)^{\frac{1}{2}} \leq C\|f\|_{L^{2}\left(\mathbb{R}^{2}\right)}
\end{aligned}
$$

Since $I^{\prime \prime}$ can be split in parts each bounded by the norms of $f$ and $g$ we may use Lebesgue's theorem about dominated convergence to obtain the claim.

By Hölder's inequality

$$
L_{2 \delta}^{p}\left(\mathbb{R}^{n}\right) \subset L_{1}^{2}\left(\mathbb{R}^{n}\right), \quad n \geq 2,
$$

where $p$ and $\delta$ are as in Theorem 1.1. Further analysis is carried out in the larger space $L_{1}^{2}\left(\mathbb{R}^{n}\right)$.

Lemma 3.4. Let $n=2$ and $f, g \in L_{1}^{2}\left(\mathbb{R}^{2}\right)$. Then $I^{\prime}$ belongs to $H^{s}\left(\mathbb{R}^{2}\right)$ with $s<2$.

Proof. We will proceed analogous to [19, Lemma 4] and [13, Proposition 3.1]. Our aim is to prove that $\widehat{I^{\prime}} \in L_{s}^{2}$. Changing first variables as $\eta \mapsto \eta-\mu$ and then taking the $n$-dimensional Fourier transform of $I^{\prime}$ in $x$ we get

$$
\widehat{I}^{\prime}(\eta)=\mathrm{p} \cdot \mathrm{v} \cdot \int_{\mathbb{R}^{2}} \frac{\chi(|\eta| / 2)}{|\eta|^{2}} \frac{\widehat{f}(\eta-\mu) \widehat{g}(\mu)}{(\eta-\mu, \mu)} \mathrm{d} \mu .
$$

Then changing variables as $\mu \mapsto \eta / 2-\mu$ yields,

$$
\begin{aligned}
\widehat{I^{\prime}}(\eta)= & \mathrm{p} \cdot \mathrm{v} \cdot \int_{\mathbb{R}^{2}} \frac{\chi(|\eta| / 2)}{|\eta|^{2}} \frac{\widehat{f}(\mu+\eta / 2) \widehat{g}(\eta / 2-\mu)}{(|\eta| / 2+|\mu|)(|\eta| / 2-|\mu|)} \mathrm{d} \mu \\
= & \left(\int_{|\mu| \leq|\eta| / 2-1}+\mathrm{p} \cdot \mathrm{v} \cdot \int_{|\eta| / 2-1 \leq|\mu| \leq|\eta| / 2+1}+\int_{|\mu| \geq|\eta| / 2+1}\right) \\
& \times \frac{\chi(|\eta| / 2)}{|\eta|^{2}} \frac{\widehat{f}(\mu+\eta / 2) \widehat{g}(\eta / 2-\mu)}{(|\eta| / 2+|\mu|)(|\eta| / 2-|\mu|)} \mathrm{d} \mu \\
= & : h_{1}(\eta)+h_{2}(\eta)+h_{3}(\eta) .
\end{aligned}
$$

It is rather easy to verify that

$$
\left|h_{1}(\eta)\right|,\left|h_{3}(\eta)\right| \leq \frac{\chi(|\eta| / 2)}{|\eta|^{3}} \int_{\mathbb{R}^{2}}|\widehat{f}(\xi)||\widehat{g}(\xi+\eta)| \mathrm{d} \xi \leq \frac{\chi(|\eta| / 2)}{|\eta|^{3}}\|\widehat{f}\|_{L^{2}\left(\mathbb{R}^{2}\right)}\|\widehat{g}\|_{L^{2}\left(\mathbb{R}^{2}\right)} .
$$


Next we turn our attention to $h_{2}$. By switching to polar coordinates the principal value integral becomes

$$
\begin{aligned}
h_{2}(\eta) & =\lim _{\varepsilon \rightarrow 0+}\left(\int_{|\eta| / 2-1 \leq r \leq|\eta| / 2-\varepsilon} r \int_{\mathbb{S}^{1}} \frac{\chi(|\eta| / 2)}{|\eta|^{2}} \frac{\widehat{f}(r \omega+\eta / 2) \widehat{g}(\eta / 2-r \omega)}{(|\eta| / 2+r)(|\eta| / 2-r)} \mathrm{d} \omega \mathrm{d} r\right. \\
& \left.+\int_{|\eta| / 2+\varepsilon \leq r \leq|\eta| / 2+1} r \int_{\mathbb{S}^{1}} \frac{\chi(|\eta| / 2)}{|\eta|^{2}} \frac{\widehat{f}(r \omega+\eta / 2) \widehat{g}(\eta / 2-r \omega)}{(|\eta| / 2+r)(|\eta| / 2-r)} \mathrm{d} \omega \mathrm{d} r\right) .
\end{aligned}
$$

Changing variables $r \mapsto|\eta|-r$ in the latter integral we obtain

$$
\begin{gathered}
h_{2}(\eta)=\lim _{\varepsilon \rightarrow 0+}\left(\int_{|\eta| / 2-1 \leq r \leq|\eta| / 2-\varepsilon} r \int_{\mathbb{S}^{1}} \frac{\chi(|\eta| / 2)}{|\eta|^{2}} \frac{\widehat{f}(r \omega+\eta / 2) \widehat{g}(\eta / 2-r \omega)}{(|\eta| / 2+r)(|\eta| / 2-r)} \mathrm{d} \omega \mathrm{d} r\right. \\
\left.-\int_{|\eta| / 2-1 \leq r \leq|\eta| / 2-\varepsilon}(|\eta|-r) \int_{\mathbb{S}^{1}} \frac{\chi(|\eta| / 2)}{|\eta|^{2}} \frac{\widehat{f}(|\eta| \omega-r \omega+\eta / 2) \widehat{g}(\eta / 2-|\eta| \omega+r \omega)}{(3|\eta| / 2-r)(|\eta| / 2-r)} \mathrm{d} \omega \mathrm{d} r\right)
\end{gathered}
$$

or

$$
\begin{array}{r}
h_{2}(\eta)=\lim _{\varepsilon \rightarrow 0+} \int_{|\eta| / 2-1 \leq r \leq|\eta| / 2-\varepsilon} \int_{\mathbb{S}^{1}} \frac{\chi(|\eta| / 2)}{|\eta|^{2}}\left(\frac{r}{(|\eta| / 2+r)(|\eta| / 2-r)}\right. \\
\left.\quad-\frac{|\eta|-r}{(3|\eta| / 2-r)(|\eta| / 2-r)}\right) \widehat{f}(r \omega+\eta / 2) \widehat{g}(\eta / 2-r \omega) \mathrm{d} \omega \mathrm{d} r \\
\quad+\lim _{\varepsilon \rightarrow 0+} \int_{|\eta| / 2-1 \leq r \leq|\eta| / 2-\varepsilon} \int_{\mathbb{S}^{1}} \frac{|\eta|-r}{(3|\eta| / 2-r)(|\eta| / 2-r)} \frac{\chi(|\eta| / 2)}{|\eta|^{2}} \\
\times[\widehat{f}(r \omega+\eta / 2) \widehat{g}(\eta / 2-r \omega)-\widehat{f}(|\eta| \omega-r \omega+\eta / 2) \widehat{g}(\eta / 2-|\eta| \omega+r \omega)] \mathrm{d} \omega \mathrm{d} r \\
=: B_{1}+B_{2} .
\end{array}
$$

Here the term $B_{1}$ can be estimated by

$$
\left|B_{1}\right| \leq \frac{\chi(|\eta| / 2)}{|\eta|^{4}}\|f\|_{L^{2}\left(\mathbb{R}^{2}\right)}\|g\|_{L^{2}\left(\mathbb{R}^{2}\right)} .
$$

In the term $B_{2}$ an elementary estimate gives

$$
\left|\frac{|\eta|-r}{(3|\eta| / 2-r)(|\eta| / 2-r)}\right| \leq \frac{r}{|\eta|} \frac{1}{\frac{|\eta|}{2}-r} .
$$

Next we use the Hajłasz inequality [9]

$$
|\varphi(x)-\varphi(y)| \leq C|x-y|[M(|\nabla \varphi|)(x)+M(|\nabla \varphi|)(y)]
$$


for $\varphi \in W_{p}^{1}\left(\mathbb{R}^{2}\right), 1<p \leq \infty$ and for almost all $x, y \in \mathbb{R}^{2}$, where

$$
M \varphi(x)=\sup _{r>0} \frac{1}{m(B(x, r))} \int_{B(x, r)}|\varphi(y)| \mathrm{d} y, \quad x \in \mathbb{R}^{n}
$$

is the Hardy-Littlewood maximal function. Here $B(x, r)$ denotes the open ball of radius $r$, centered at $x$ with Lebesgue measure $m(B(x, r))$. Recall that $M: L^{p} \rightarrow L^{p}$, $1<p \leq \infty$, see e.g., [20]. For this purpose we expand

$$
\begin{aligned}
\widehat{f}(r \omega+\eta / 2) \widehat{g}(\eta / 2-r \omega) & -\widehat{f}(|\eta| \omega-r \omega+\eta / 2) \widehat{g}(\eta / 2-|\eta| \omega+r \omega) \\
= & \widehat{f}(r \omega+\eta / 2)[\widehat{g}(\eta / 2-r \omega)-\widehat{g}(\eta / 2-|\eta| \omega+r \omega)] \\
& \quad+\widehat{g}(\eta / 2-|\eta| \omega+r \omega)[\widehat{f}(r \omega+\eta / 2)-\widehat{f}(|\eta| \omega-r \omega+\eta / 2)] .
\end{aligned}
$$

In the next step we find it convenient to slightly expand the region of integration. Now, since $f, g \in L_{1}^{2}\left(\mathbb{R}^{2}\right)$ then $\widehat{f}, \widehat{g} \in H^{1}\left(\mathbb{R}^{2}\right)$ and we have

$$
\begin{aligned}
\left|B_{2}\right| & \leq \frac{\chi(|\eta| / 2)}{|\eta|^{3}} \int_{\frac{|\eta|}{2}-1 \leq|\xi| \leq \frac{|\eta|}{2}+1}|\widehat{f}(\eta / 2+\xi)| \mid M(\nabla \widehat{g})(\eta / 2-\xi) \mathrm{d} \xi \\
& +\frac{\chi(|\eta| / 2)}{|\eta|^{3}} \int_{\frac{|\eta|}{2}-1 \leq|\xi| \leq \frac{|\eta|}{2}+1}|\widehat{f}(\eta / 2+\xi)| \mid M(\nabla \widehat{g})(\eta / 2-|\eta| \widehat{\xi}+\xi) \mathrm{d} \xi \\
& +\frac{\chi(|\eta| / 2)}{|\eta|^{3}} \int_{\frac{|\eta|}{2}-1 \leq|\xi| \leq \frac{|\eta|}{2}+1}|\widehat{g}(\eta / 2-|\eta| \widehat{\xi}+\xi)| \mid M(\nabla \widehat{f})(\eta / 2+\xi) \mathrm{d} \xi \\
& +\frac{\chi(|\eta| / 2)}{|\eta|^{3}} \int_{\frac{|\eta|}{2}-1 \leq|\xi| \leq \frac{|\eta|}{2}+1}|\widehat{g}(\eta / 2-|\eta| \widehat{\xi}+\xi)| \mid M(\nabla \widehat{f})(\eta / 2+|\eta| \widehat{\xi}-\xi) \mathrm{d} \xi \\
& =: J_{1}+J_{2}+J_{3}+J_{4} .
\end{aligned}
$$

Here the hat-notation is used for two meanings: hat on a function denotes the usual $n$-dimensional Fourier transform, while a hat on a vector $\xi \in \mathbb{R}^{n}$ is used to denote the unit vector $\widehat{\xi}:=\xi /|\xi|$. The first integral above is easily estimated by Cauchy-Schwarz inequality as

$$
J_{1} \leq \frac{C \chi(|\eta| / 2)}{|\eta|^{3}}\|f\|_{L^{2}\left(\mathbb{R}^{2}\right)}\|M(\nabla \widehat{g})\|_{L^{2}\left(\mathbb{R}^{2}\right)} .
$$

In the integrals $J_{2}, J_{3}$ and $J_{4}$ we first use the Cauchy-Schwarz inequality and then change variables as follows. We consider an orthogonal reflection $\varphi_{\eta}(\xi)=|\eta| \widehat{\xi}-\xi$ in the annulus $A_{\eta}:=\left\{\xi \in \mathbb{R}^{2}|| \eta|/ 2-1 \leq| \xi|\leq| \eta \mid / 2+1\right\}$ with respect to the circle of radius $|\eta| / 2$ centered at origin. This mapping $\varphi_{\eta}: A_{\eta} \rightarrow A_{\eta}$ is bijective with $\varphi_{\eta}=\varphi_{\eta}^{-1}$ and has Jacobian

$$
J_{\varphi}=1-\frac{|\eta|}{|\xi|},
$$


which is estimated by $\frac{1}{3} \leq\left|J_{\varphi}\right| \leq 3$ within the domain of integration (we take $k_{0}>2$ to obtain $|\eta|>4$ ). This procedure yields for the term $h_{2}$ the estimate

$$
\left|h_{2}\right| \leq C \frac{\chi(|\eta| / 2)}{|\eta|^{3}}\left(\|f\|_{L^{2}\left(\mathbb{R}^{2}\right)}\|M(\nabla \widehat{g})\|_{L^{2}\left(\mathbb{R}^{2}\right)}+\|g\|_{L^{2}\left(\mathbb{R}^{2}\right)}\|M(\nabla \widehat{f})\|_{L^{2}\left(\mathbb{R}^{2}\right)}\right) .
$$

These estimates show that $\widehat{I^{\prime}}$ belongs to $L_{s}^{2}\left(\mathbb{R}^{2}\right)$ with $s<2$ which then implies the claim.

\subsection{Three-dimensional case}

In this section we consider the first non-linear term $q_{1}$ for $n=3$. For simplicity, we assume that the coefficients $\vec{q}$ and $V$ are real-valued. Next we define the solutions to the scattering problem (3) for $k<0$ by requiring $u(x, k, \theta)=\overline{u(x,-k, \theta)}$ and extend the scattering amplitude by $A\left(k, \theta, \theta^{\prime}\right)=\overline{A\left(-k, \theta, \theta^{\prime}\right)}$ for $k<0$.

It follows from (8) that

$$
\begin{aligned}
\operatorname{Re} q_{\mathrm{B}}(x) & =\frac{1}{(2 \pi)^{n}} \operatorname{Re}\left(\int_{0}^{\infty} k^{n-1} \int_{\mathbb{S}^{n-1}} \mathrm{e}^{-\mathrm{i} k(\theta, x)} A_{\mathrm{b}}\left(\frac{k}{2}, \theta\right) \mathrm{d} \theta \mathrm{d} k\right) \\
& =\frac{1}{2(2 \pi)^{n}} \int_{-\infty}^{\infty}|k|^{n-1} \int_{\mathbb{S}^{n-1}} \mathrm{e}^{-\mathrm{i} k(\theta, x)} A_{\mathrm{b}}\left(\frac{k}{2}, \theta\right) \mathrm{d} \theta \mathrm{d} k .
\end{aligned}
$$

Remark 3.5. Sometimes (11) is taken as the definition of the backscattering Born approximation (see e.g., $[14,19])$. However, the definition (8) is more general since it allows the approximation of complex-valued $\beta$.

In the splitting

$$
q_{\mathrm{B}}=\beta+\widetilde{q}+q_{1, M}+q_{1, E}+q_{\mathrm{rest}}
$$

we have already studied the terms $q_{1, E}$ and $q_{\text {rest }}$. So it remains to examine

$$
\operatorname{Re} q_{1, M}(x)=\frac{1}{2(2 \pi)^{n}} \int_{-\infty}^{\infty}|k|^{n-1} \int_{\mathbb{S}^{n-1}} \mathrm{e}^{-\mathrm{i} k(\theta, x)} A_{\mathrm{M}}\left(\frac{k}{2}, \theta\right) \mathrm{d} \theta \mathrm{d} k=: q_{1, \mathrm{M}}^{+}(x)+q_{1, \mathrm{M}}^{-}(x),
$$

where $q_{1, \mathrm{M}}^{+}$and $q_{1, \mathrm{M}}^{-}$correspond to integrals over positive and negative $k$, respectively.

Lemma 3.6. Let $n \geq 2$. If $\vec{q}$ and $V$ are real-valued and if they are as in Theorem 
1.1, then

$$
\begin{aligned}
q_{1, \mathrm{M}}^{ \pm} & =\frac{(2 \pi)^{\frac{n}{2}}}{4} \mathcal{F}^{-1}\left(\frac{\chi(|\mu+\eta| / 2)}{|\mu+\eta|^{2}} \frac{\widehat{\nabla \cdot \vec{q}}(\eta) \widehat{\nabla \cdot \vec{q}}(\mu)}{(\mu, \eta) \pm \mathrm{i} 0}\right)(x, x) \\
& -\frac{(2 \pi)^{\frac{n}{2}}}{4} \mathcal{F}^{-1}\left(\frac{\chi(|\mu+\eta| / 2)}{|\mu+\eta|^{2}} \frac{\sum_{j, k=1}^{n} \widehat{\partial_{j} q_{k}}(\eta) \widehat{\partial_{k} q_{j}}(\mu)}{(\mu, \eta) \pm \mathrm{i} 0}\right)(x, x) \\
& -(2 \pi)^{\frac{n}{2}} \mathcal{F}^{-1}\left(\frac{\chi(|\mu+\eta| / 2)}{|\mu+\eta|^{2}} \frac{\widehat{\widetilde{V}}(\eta) \widehat{V}(\mu)}{(\mu, \eta) \pm \mathrm{i} 0}\right)(x, x)
\end{aligned}
$$

in the sense of distributions.

Proof. Literally the same as the proof of Lemma 3.2, but for the integral over negative $k$ we use the definition $A_{\mathrm{M}}(k, \theta)=\overline{A_{\mathrm{M}}(-k, \theta)}$. It is at this complex conjugation where the assumptions $\vec{q}=\overline{\vec{q}}$ and $V=\bar{V}$ are used.

Using the fact that $\vec{q}$ and $V$ are real-valued, (10) provides a convenient cancellation of delta terms and it suffices to study

$$
I=\text { p. v. } \int_{\mathbb{R}^{n} \times \mathbb{R}^{n}} \mathrm{e}^{\mathrm{i}(x, \eta+\mu)} \frac{\chi(|\mu+\eta| / 2)}{|\mu+\eta|^{2}} \frac{\widehat{f}(\eta) \widehat{g}(\mu)}{(\eta, \mu)} \mathrm{d} \eta \mathrm{d} \mu .
$$

Lemma 3.7. Let $n=3$ and $f, g \in L_{1}^{2}\left(\mathbb{R}^{3}\right)$. Then $I$ belongs to $H^{s}\left(\mathbb{R}^{3}\right)$ with $s<3 / 2$.

Proof. The claim is obtained by repeating the proof of Lemma 3.4 with minor changes. The only relevant difference occurs when changing to polar coordinates in the term $h_{2}(\eta)$, where the Jacobian brings forth an $r^{2}$-multiplier. Fortunately this fact does not affect any subsequent estimates and the proof can be carried out in the same way as before.

\section{Main results}

Theorem 4.1 (Main theorem, $n=2$ ). Let $\vec{q} \in W_{p, 2 \delta}^{1}\left(\mathbb{R}^{2}\right)$ and $V \in L_{2 \delta}^{p}\left(\mathbb{R}^{2}\right)$, where $2<p \leq \infty$ and $2 \delta>2-\frac{2}{p}$. Then the difference $q_{\mathrm{B}}-\beta$ defines a bounded and continuous function. If in addition $\vec{q}$ and $V$ are real-valued then $\operatorname{Re} q_{\mathrm{B}}-\beta \in H^{s}\left(\mathbb{R}^{2}\right)$ $\left(\bmod C^{\infty}\left(\mathbb{R}^{2}\right)\right)$ for any $s<2$.

Proof. Since

$$
q_{\mathrm{B}}-\beta=q_{1, \mathrm{M}}+q_{1, \mathrm{E}}+q_{\mathrm{rest}}+\widetilde{q}
$$

then the first claim follows from Lemmata 2.1, 3.1, 3.3 and 3.4. For real-valued potentials the second claim is based on the fact that $I^{\prime \prime}=0$. 
Corollary 4.2. If $\vec{q}$ and $V$ are as in Theorem 4.1 then the jumps of $\beta$ over smooth (bounded) curves are uniquely determined by the backscattering data $A_{\mathrm{b}}$ and can be recovered from $q_{\mathrm{B}}$.

Theorem 4.3 (Main theorem, $n=3$ ). Let $\vec{q} \in W_{p, 2 \delta}^{1}\left(\mathbb{R}^{3}\right)$ and $V \in L_{2 \delta}^{p}\left(\mathbb{R}^{3}\right)$, where $3<p \leq \infty$ and $2 \delta>3-\frac{3}{p}$, be real-valued. Then the difference $\operatorname{Re} q_{\mathrm{B}}-\beta$ belongs to the Sobolev space $H^{s}\left(\mathbb{R}^{3}\right)\left(\bmod C^{\infty}\left(\mathbb{R}^{3}\right)\right)$ for any $s<\frac{3}{2}$.

Proof. The proof follows from Lemmata 2.1, 3.1 and 3.7.

Corollary 4.4. If $\vec{q}$ and $V$ are as in Theorem 4.3 then the main singularities of $\beta$ over smooth (bounded) curves are uniquely determined by the backscattering data $A_{\mathrm{b}}$ and can be recovered from $q_{\mathrm{B}}$.

\section{$5 \quad$ Numerics}

In this section we present some numerical examples to illustrate the recovery of singularities using the inverse Born approximation in two dimensions. We proceed analogously to $[6,10]$ but outline the numerical scheme here for convenience.

We use synthetic scattering data computed from

$$
A(k, \theta,-\theta) \approx-\frac{4 \sqrt{2 \pi R}}{\mathrm{ie}^{-\mathrm{i} \pi / 4}} \frac{1}{k^{-5 / 2} \mathrm{e}^{\mathrm{i} k R}} u_{\mathrm{sc}}(-R \theta, k, \theta), \quad R=10^{5} .
$$

To this end, we need an approximation for $u_{\mathrm{sc}}$. That is computed as the truncated series $u_{\mathrm{sc}} \approx \sum_{j=1}^{M} u_{j}$ with $M=2$. The integrals $u_{j}$ are calculated numerically over the supports of $\vec{q}$ and $V$.

Having the backscattering amplitude at hand the inverse Born approximation is computed from (8). Indeed, we represent $q_{\mathrm{B}}(x)$ in discrete form

$$
q_{\mathrm{B}}(x)=\sum_{j=1}^{N} f_{j} \chi_{r_{j}}(x),
$$

where $r_{j}$ are the subgrids of unit cube in $\mathbb{R}^{2}$ and $f_{j}$ are the unknown values of $q_{\mathrm{B}}$ at those subgrids. Substituting this representation into

$$
\int_{\mathbb{R}^{n}} \mathrm{e}^{\mathrm{i} k(\theta, x)} q_{\mathrm{B}}(x) \mathrm{d} x=A\left(k, \theta, \theta^{\prime}\right)
$$

yields

$$
\sum_{j=1}^{N} f_{j} \int_{r_{j}} \mathrm{e}^{\mathrm{i} k(\theta, x)} \mathrm{d} x=A\left(k, \theta, \theta^{\prime}\right),
$$


where the integrals are easy to compute in closed form. Evaluating the latter equation at several $k=k_{s}, \theta=\theta_{l}$ points leads to a linear system which we write as $E f=g$. We use the following parameter values:

$$
N=6400, \quad k=k_{s}=s, \quad s=1,2, \ldots, 12
$$

and 40 values for $\theta$ uniformly from the unit circle.

The right hand side $g$ is corrupted with zero mean Gaussian noise with standard deviation $1 \%$ of maximum value. The ill-posed linear system is solved for $f$ using a regularization method. Namely, we use the truncated singular value decomposition (TSVD) as follows. Let $E=U S V^{*}$ be the singular value decomposition of $E$, where $S=\operatorname{diag}\left(s_{1}, \ldots, s_{r}\right)$ is the diagonal matrix containing the singular values $s_{j}$ of $E$. Then the reconstruction is

$$
f=V L U^{*} g,
$$

where $L=\operatorname{diag}\left(1 / s_{1}, \ldots, 1 / s_{\text {tol }}, 0, \ldots, 0\right)$ and where $s_{\text {tol }}$ is the last (smallest) singular value exceeding a prescribed tolerance $10^{-2}$.

We consider the following sample scatterers:

$$
V(x)=\frac{3}{2} \chi_{\Omega_{V}}(x)
$$

where $\Omega_{V}$ is an ellipse (Example 1) or rectangle (Examples 2 and 3 ). For $q_{j}, j=1,2$ we consider the smooth bump function

$$
\frac{1}{2} \chi_{|x|<1}(x) \exp \left(1 /\left(|x|^{2}-1\right)\right)
$$

and scale the variables so that the supports $\Omega_{j}:=\operatorname{supp} q_{j}$ are ellipses. Further, in Example 3 we multiply the potentials $V$ and $q_{2}$ by the imaginary unit i to illustrate the recovery of complex-valued potentials.

Figure 1 shows the contour plots of $V$ and $\vec{q}$ with white lines indicating their supports. The shape and location of real and imaginary parts of the potentials in Example 3 are the same as for the real-valued potentials in Example 2. Figures 2 and 3 present the unknown function $\beta$ for Examples 1 and 2 on the left and their TSVD reconstructions on the right. These figures show that by using the inverse Born backscattering approximation we are able to locate quite accurately the shape and location of the support of the scatterers. Additionally, the height function of $\beta$ is recovered rather reasonably. Figure 4 presents the real and imaginary parts of potentials in Example 3 on the left with their respective TSVD reconstructions on the right. In light of this example we see that the Born approximation can be applied numerically also in the complex-valued case. 

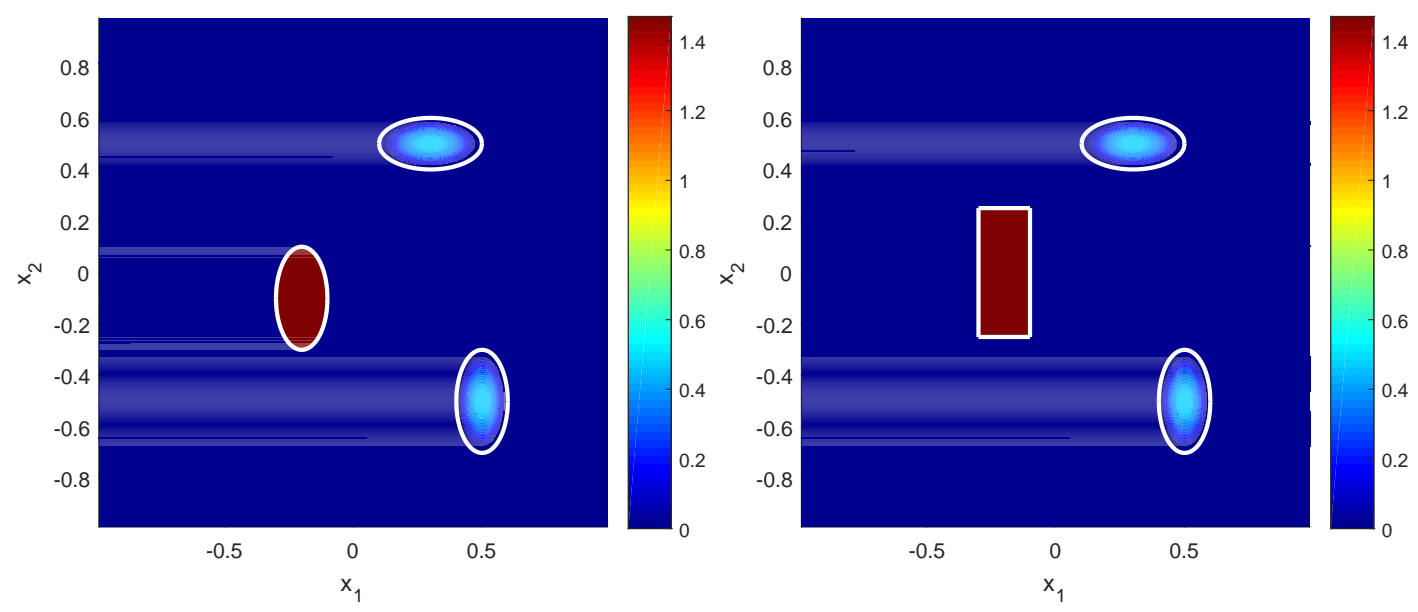

Figure 1: Potentials $V$ and $\vec{q}$ for sample scatterers: Example 1 (left) and Example 2 (right)
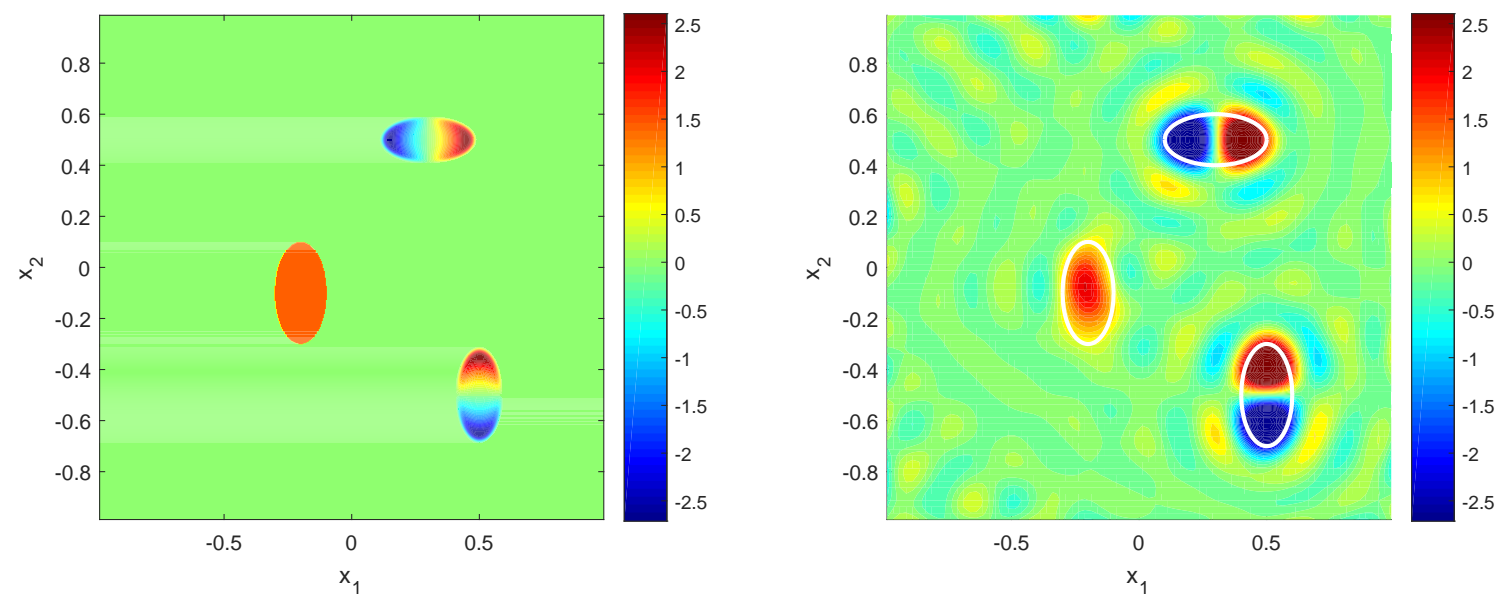

Figure 2: Example 1: Function $\beta(x)$ (left) and TSVD reconstruction (right)

\section{Conclusions}

An inverse backscattering problem for the first order perturbation of the multidimensional biharmonic operator was studied. We examined the problem of finding the jump discontinuities and local singularities of the potential functions based on certain scattering data. This data for the inverse problem is so-called scattering amplitude, which can be obtained as the far-field data or, more precisely, as the asymptotic of the scattered wave at $|x| \rightarrow \infty$ [24]. In this text we assumed that only part of the full data is known; namely, we assumed that the scattering amplitude is only known for arbitrarily large wave-numbers $k$ and for measurement angles 

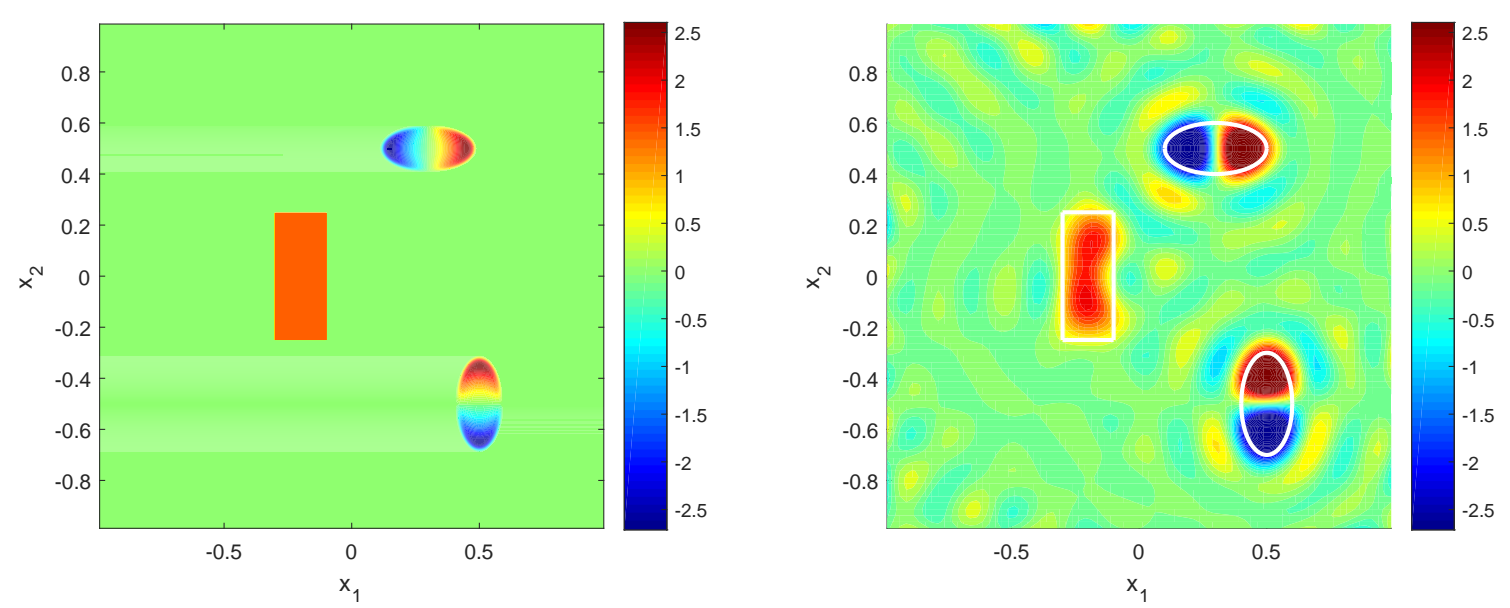

Figure 3: Example 2: Function $\beta(x)$ (left) and TSVD reconstruction (right)

which are opposite to the angle of the incident wave.

The method of choice, backscattering Born approximation, was studied separately for the dimensions $n=2$ and $n=3$ of the ambient space. It was proved that backscattering Born approximation can be used to recover the jumps and singularities of a combinations of the (possibly complex-valued) potentials in two dimensions, and to recover main singularities in three dimensions. Numerical examples were given to further illustrate this approximation method for several different scatterers, including a complex-valued example.

\section{Acknowledgement}

This work was supported by the Academy of Finland (application number 250215, Finnish Programme for Centres of Excellence in Research 2012-2017). The first author was supported by the Doctoral Programme of Exact Sciences at the University of Oulu, Finland.

\section{References}

[1] Aktosun T and Papanicolaou V G, Time evolution of the scattering data for a fourth-order linear differential operator, Inverse Problems, 24 (2008), 055013.

[2] Choudhury A and Heck H, Stability of the inverse boundary value problem for the biharmonic operator: Logarithmic estimates, Journal of Inverse and Illposed Problems, 25(2), 2016, 251-263. 

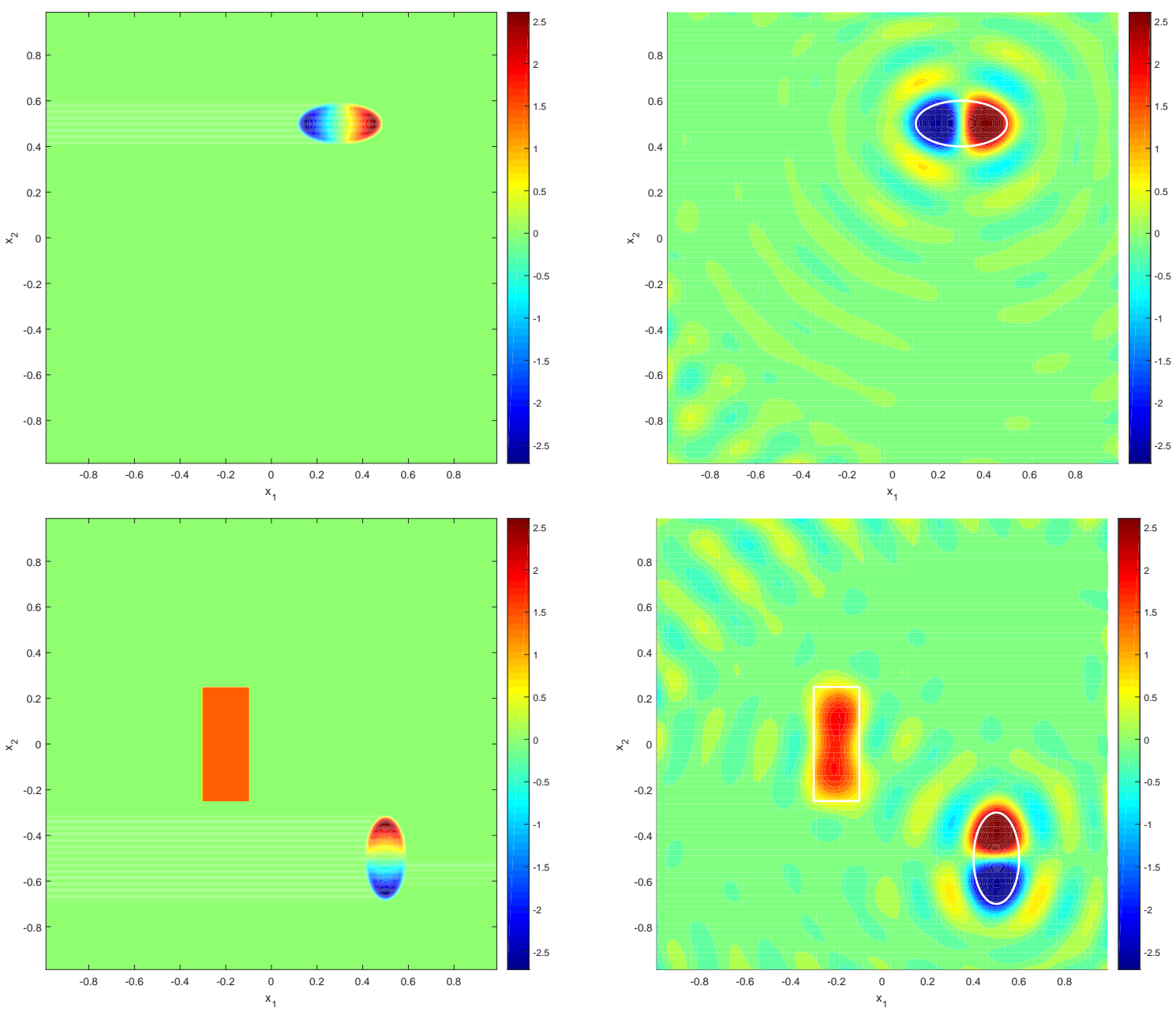

Figure 4: Example 3: real and imaginary parts of $\beta$ (top- and bottom-left) and real and imaginary parts of TSVD reconstruction (top- and bottom-right)

[3] Eskin G and Ralston J, The Inverse Backscattering Problem in Three Dimensions, Commun. Math. Phys. 124 (1989), 169-215.

[4] Eskin G and Ralston J, Inverse Backscattering in Two Dimensions, Commun. Math. Phys. 138 (1991),451-486.

[5] Eskin G and Ralston J, Inverse Scattering Problem for the Schrödinger Equation with Magnetic Potential at a Fixed Energy, Commun. Math. Phys. 173 (1995), 199-224.

[6] Fotopoulos G and Harju M, Inverse scattering with fixed observation angle data in 2D, Inverse Problems in Science and Engineering 2X (2017). 
[7] Fotopoulos G, Harju M and Serov V, Inverse fixed angle scattering and backscattering for a nonlinear Schrödinger equation in 2D, Inverse Problems and Imaging, 7 (2013), 183-197.

[8] Gazzola F, Grunau H C and Sweers G, Polyharmonic Boundary Value Problems. Springer-Verlag Berlin Heidelberg, 2010.

[9] Hajłasz P, Sobolev spaces on an arbitrary metric space, Potential Analysis 5 (1996), 403-415.

[10] Harju M, Numerical computation of the inverse Born approximation for the nonlinear Schrödinger equation in two dimensions, Computational Methods in Applied Mathematics 16:1 (2016), 133-143.

[11] Iwasaki K, Scattering theory for 4th order differential operators: I-II, Japan. J. Math., 14 (1988),1-96.

[12] Kanwal R, Generalized functions: Theory and applications, third edition, Birkhäuser, Boston, 2004.

[13] Ola P, Päivärinta L and Serov V, Recovering singularities from backscattering in two dimensions, Communications in Partial Differential Equations, 26 (2001), 697-715.

[14] Päivärinta L and Serov V, Recovery of jumps and singularities in the multidimensional Schrödinger operator from limited data, Inverse Problems and Imaging, Volume 1, No. 3, (2007), 525-535.

[15] Reyes J M, Inverse backscattering for the Schrödinger equation in 2D, Inverse Problems 23 (2007), 625-643.

[16] Ruiz A, Recovery of the singularities of a potential from fixed angle scattering data, Comm. Partial Diff. Equ., 26:9-10 (2001), 1721-1738.

[17] Ruiz A and Vargas A, Partial Recovery of a Potential from Backscattering Data, Communications in Partial Differential Equations, 30 (2005), 67-96.

[18] Serov V, Inverse backscattering Born approximation for a two-dimensional magnetic Schrödinger operator, Inverse Problems 29:7 (2013), 075015.

[19] Serov V and Sandhu J, Inverse backscattering problem for the generalized nonlinear Schrödinger operator in two dimensions, J. Phys. A: Math. Theor. 43 (2010), 325206. 
[20] Stein E, Singular Integrals and Differentiability Properties of Functions, Princeton University Press, 1970.

[21] Sun Z and Uhlmann G, Recovery of Singularities for Formally Determined Inverse Problems, Commun. Math. Phys. 153 (1993), 431-445.

[22] Sun Z and Uhlmann G, Inverse Scattering for singular potentials in two dimensions, Trans. Amer. Math. Soc. 338 (1993).

[23] Tyni T, Harju M and Serov V, Recovery of singularities in a fourth-order operator on the line from limited data, Inverse Problems 32 (2016), 075001.

[24] Tyni T and Serov V, Scattering problems for perturbations of the multidimensional biharmonic operator, submitted, 2017.

[25] Yang Y Determining the first order perturbation of a bi-harmonic operator on bounded and unbounded domains from partial data, J. Differential Equations 257 (2014) 10, 3607-3639. 